\title{
Pollen morphology of Centaurea L. (Asteraceae) in Iran
}

\author{
Elaheh Sadat Bagheri Shabestari ${ }^{1,3}$, Farideh Attar ${ }^{2}$, Hosein Riahi $^{1}$ and Masoud Sheidai ${ }^{1}$
}

Received: 10 December, 2012. Accepted: 6 June, 2013

\begin{abstract}
The genus Centaurea $\mathrm{L}$. is one of the largest genera in the family Asteraceae. The number of taxa included in this genus ranges from 500 to 600 worldwide. Iran is one of the major centers of diversity for this genus. Pollen morphology has proved useful in the systematics of Asteraceae, as well as in that of some of its genera and species. The pollen morphology of 19 taxa of the genus Centaurea was investigated in detail by light and scanning electron microscopy. Examination under light microscopy showed pollen grains to be tricolporate, isopolar, radially symmetrical, oblate spheroidal, prolate spheroidal, subprolate, tectum perforate and scabrate. Based on the exine ornamentation observed under scanning electron microscopy, we identified two types of pollen grains, designated type I (dense acute spinules) and type II (sparse spinules). All of the taxa evaluated resemble Wagenitz's Jacea type. We present micrographs of pollen grains, together with descriptions of the characteristics of their structure. We found that the pollen characters (shape, exine structure, sculpturing) were useful for classification.
\end{abstract}

Key words: Centaurea, palynology, exine ornamentation, light microscopy, scanning electron microscopy

\section{Introduction}

The genus Centaurea L. (Asteraceae) is represented by 500-600 taxa worldwide (Rendle 1976; Heywood 1979; Hickey \& King 1981) and occurs primarily in Asia, Europe, North Africa and North America (Davis et al. 1988). In Iran, the genus is highly (42.35\%) endemic (Wagenitz 1980). Of the 86 Centaurea taxa in Iran, 36 are endemic to the country (Ghahreman \& Attar 1999). The high level of endemism of Centaurea in Iran supports the assertion that the country is one of the major centers of diversity for the genus (Wagenitz 1986).

Pollen morphology has been proved to be useful in the systematics of the Asteraceae family, as well as that of some of its genera and species (Moore et al. 1991). Wagenitz (1955) divided the genus Centaurea into eight subgenera, on the basis of exine structure and sculpturing: Serratula, Centaurium, Scabiosa, Crupina, Jacea, Dealbata, Montana and Cyanus. Villodre \& Garcia-Jacas (2000) stated that pollen type is one of the most reliable characteristics for establishing phylogeny in the Jacea group. There have been only a few studies employing light microscopy (LM) or scanning electron microscopy (SEM) to evaluate the pollen morphology of Centaurea species (Uysal et al. 2005; Celik et al. 2005; Celik et al. 2008; Özler et al. 2009; Jafari
\& Ghanbarian 2007), none of which were conducted in Iran. Therefore, the present survey aims to present detailed quantitative and qualitative data on the pollen morphology of the genus, as well as to evaluate the taxonomic value of those data.

\section{Material and methods}

We employed LM and SEM in order to conduct palynological studies of 19 species of the genus Centaurea belonging to four sections (Wagenitz 1980):

a) Phaeopappus - C. spectabilis (Fisch. \& C.A. Mey) Schultz-Bip., C. aucheri subsp. aucheri, C. aucheri subsp. szowitsii (Boiss.) Wagenitz, C. aucheri subsp. indistincta Wagenitz, C. aucheri subsp. elbursensis Wagenitz. C. aucheri subsp. farsistanica Wagenitz and C. albonitens Turill.

b) Cynaroides - C. regia subsp. regia, Boiss. C. imperialis Hausskn. ex Bornm., C. phlomoides Boiss. \& Hausskn., C. geluensis Boiss. \& Hausskn. and C. gabrielae (Bornm.) Wagenitz.

c) Paraphysis - including C. amadanensis var. amadanensis, C. amadanensis var. gymnoclada (Jaub. \& Spach.) Wagenitz and C. nemecii Nab.

d) Microlophus - C. polypodiifolia var. polypodiifolia, C. behen L., C. pabotii Wagenitz and C. koieana Bornm.

\footnotetext{
${ }^{1}$ Shahid Beheshti University, GC, Faculty of Biological Sciences, Tehran, Iran

${ }^{2}$ University of Tehran, School of Biology and Center of Excellence in Phylogeny of Living Organisms, College of Science, Tehran, Iran

${ }^{3}$ Author for correspondence: bagheri.elaheh@gmail.com
} 
Voucher specimens are listed in Table 1. Voucher specimens are deposited in the Central Herbarium of Tehran University (code, TUH), the Herbarium of the Iranian Research Institute of Plant Protection (code, IRAN) and the Herbarium of Shahid Beheshti University (code, HSBU). We studied a total of 15 quantitative and qualitative pollen characters (Table 2-4). Characters were selected on the basis of those reported in the literature (Özler et al. 2009; Zamani et al. 2010) and our own observations of Centaurea species. Pollen obtained from flower buds at anthesis was prepared for LM according to Wodehouse (1935) and Erdtman (1960). The LM observations of acetolyzed pollen grains were made with an Olympus binocular microscope at 1000× magnification. Acetolyzed pollen grains were observed and measured with ImageTool software, version 3 (UTHSCSA, Texas, USA). Measurements are scored for at least 20-25 mature pollen grains per specimen.

For SEM, after acetolysis, the specimens were mounted on $12.5 \mathrm{~mm}$ diameter aluminum stubs and sputter-coated with approximately $25 \mathrm{~nm}$ of gold-palladium. The specimens were examined and photographed with a scanning electron microscope (XL30; Philips, Eindhoven, The Netherlands) at an accelerating voltage of $20 \mathrm{kV}$. The terminology follows mainly Ertdman (1952) and Halbitter et al. (2007). We analyzed a total of 19 taxa belonging to the genus Centaurea. Among such taxa, the exine sculpture is reportedly scabrate when the sculpturing elements are less than $1 \mu \mathrm{m}$ and microechinate when they are 1-2 $\mu \mathrm{m}$ (Wagenitz 1955).

\section{Results}

All of the morphological parameters investigated are shown in table 2-4 and in fig. 1-75. Under LM, the pollen grains are isopolar, radially symmetrical, oblate spheroidal, prolate spheroidal and subprolate. Their apertures are tricolporate. In the polar view, pollen grains are circular and the colpi are acute at both ends (Fig. 58-75). The colpus membrane is granulate-perforate. In all taxa examined, the ectoaperture is bigger than the endoaperture (6.96-18.66 $\mu \mathrm{m}$ vs. 2.93-7.73 $\mu \mathrm{m}$ ) (Table 3).

The distance between colpi ranged from $22.35 \pm 1.31 \mu \mathrm{m}$ (Table 3), in C. koieana, to $37.11 \pm 2.09 \mu \mathrm{m}$, in C. amadanensis var. amadanensis. The distance between a colpus and the pole was longest for C. aucheri subsp. aucheri $(22.27 \pm 1.49$ $\mu \mathrm{m})$ and shortest for $C$. pabotii $(13.37 \pm 1.02 \mu \mathrm{m})$.

The polar diameter ranges from $39.98 \pm 1.09$ (Table 2), in Centaurea behen, to $55.37 \pm 2.05 \mu \mathrm{m}$, in C. aucheri subsp. aucheri; the equatorial diameter ranges from $34.69 \pm 1.26$, in C. koieana, to $49.03 \pm 1.63 \mu \mathrm{m}$, in C. aucheri subsp. aucheri.

Under LM and SEM, the exine sculpture is scabrate and the tectum is perforate, respectively (Fig. 1-57). Exine thickness ranges from $4.52 \mu \mathrm{m}$, in Centaurea koieana, to $7.81 \mu \mathrm{m}$, in C. gabrielae. Under SEM, the number of perforations counted ranged from $19.71 / \mu \mathrm{m}^{2}$, in C. aucheri subsp. indistincta, to $5.04 / \mu^{2}$, in C. polypodiifolia var. polypodiifolia. The number of microspines ranged from $5.5 / \mu \mathrm{m}^{2}$, in

Table 1. List of taxa of Centaurea L. (Asteraceae) examined, collectors and herbarium number.

\begin{tabular}{|c|c|c|}
\hline Taxa & Date & Collector, herbarium-accession number \\
\hline C. albonitens & 20 June, 1982 & Ghahreman et al., TUH-11447 \\
\hline C. amadanensis var. amadanensis & 7 June, 2009 & Bagheri, HSBU-1007 \\
\hline C. amadanensis var. gymnoclada & 8 July, 1995 & Ghahreman \& Attar, TUH-29650 \\
\hline C. aucheri subsp. aucheri & 11 June, 2009 & Bagheri, HSBU-1002 \\
\hline C. aucheri subsp. elbursensis & 28 May, 2009 & Mehrabian, HSBU-1004 \\
\hline C. aucheri subsp. farsistanica & 25 June, 1987 & Kashkuli, IRAN-7913 \\
\hline C. aucheri subsp. indistincta & 28 May, 1968 & Termeh, IRAN-7932 \\
\hline C. aucheri subsp. szowitsii & 14 June, 2009 & Bagheri, HSBU-1003 \\
\hline C. behen & 11 July, 2009 & Bagheri, HSBU-1005 \\
\hline C. gabrielae & 27 April, 1983 & Termeh et al., IRAN-842 \\
\hline C. geluensis & 23 June, 1999 & Veiskarami, TUH-23715 \\
\hline C. imperialis & 9 July, 1995 & Ghahreman \& Attar, TUH-19667 \\
\hline C. koieana & 3 June, 1999 & Ghahreman et al., TUH-22380 \\
\hline C. nemecii & 13 June, 1988 & Ghaffari, TUH-5867 \\
\hline C. pabotii & 7 June, 2009 & Bagheri, HSBU-1006 \\
\hline C. phlomoides & 3 July, 1996 & Ghahreman \& Attar, TUH-19857 \\
\hline C. polypodiifolia var. polypodiifolia & 27 June, 1994 & Ghahreman \& Mozaffarian, TUH-17438 \\
\hline C. regia subsp. regia & 3 July, 1996 & Ghahreman \& Attar, TUH-20565 \\
\hline C. spectabilis & 9 June, 2010 & Bagheri, HSBU-1001 \\
\hline
\end{tabular}

HSBU - Herbarium of Shahid Beheshti University; TUH - Central Herbarium of Tehran University; IRAN - Herbarium of the Iranian Research Institute of Plant Protection. 

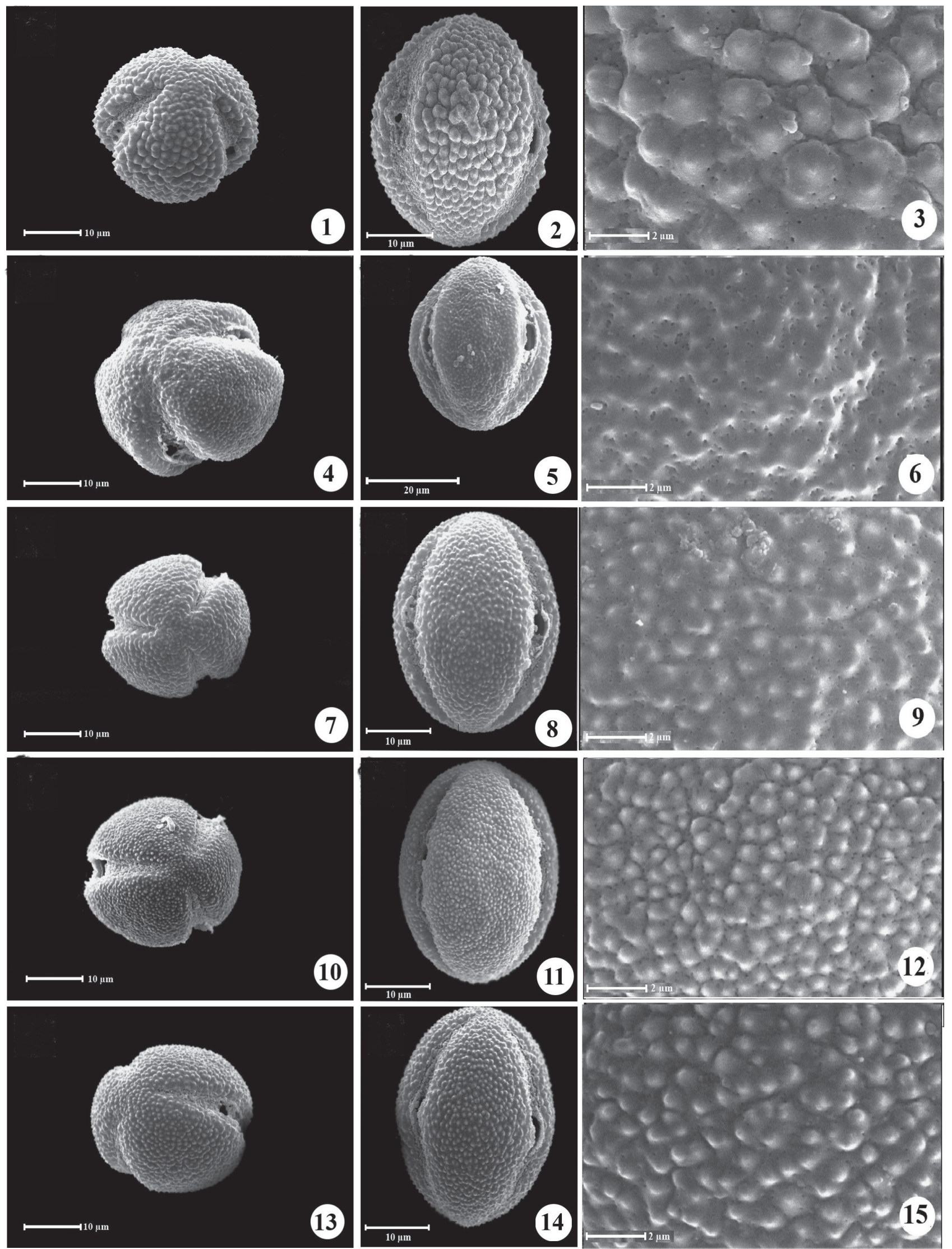

Figures 1-15. Pollen grains (SEM) of species of Centaurea L. (Asteraceae). (1-3) C. albonitens; (4-6) C. aucheri subsp. aucheri; (7-9) C. aucheri subsp. elbursensis; (10-12) C. aucheri subsp. farsistanica; (13-15) C. aucheri subsp. szowitsii. 

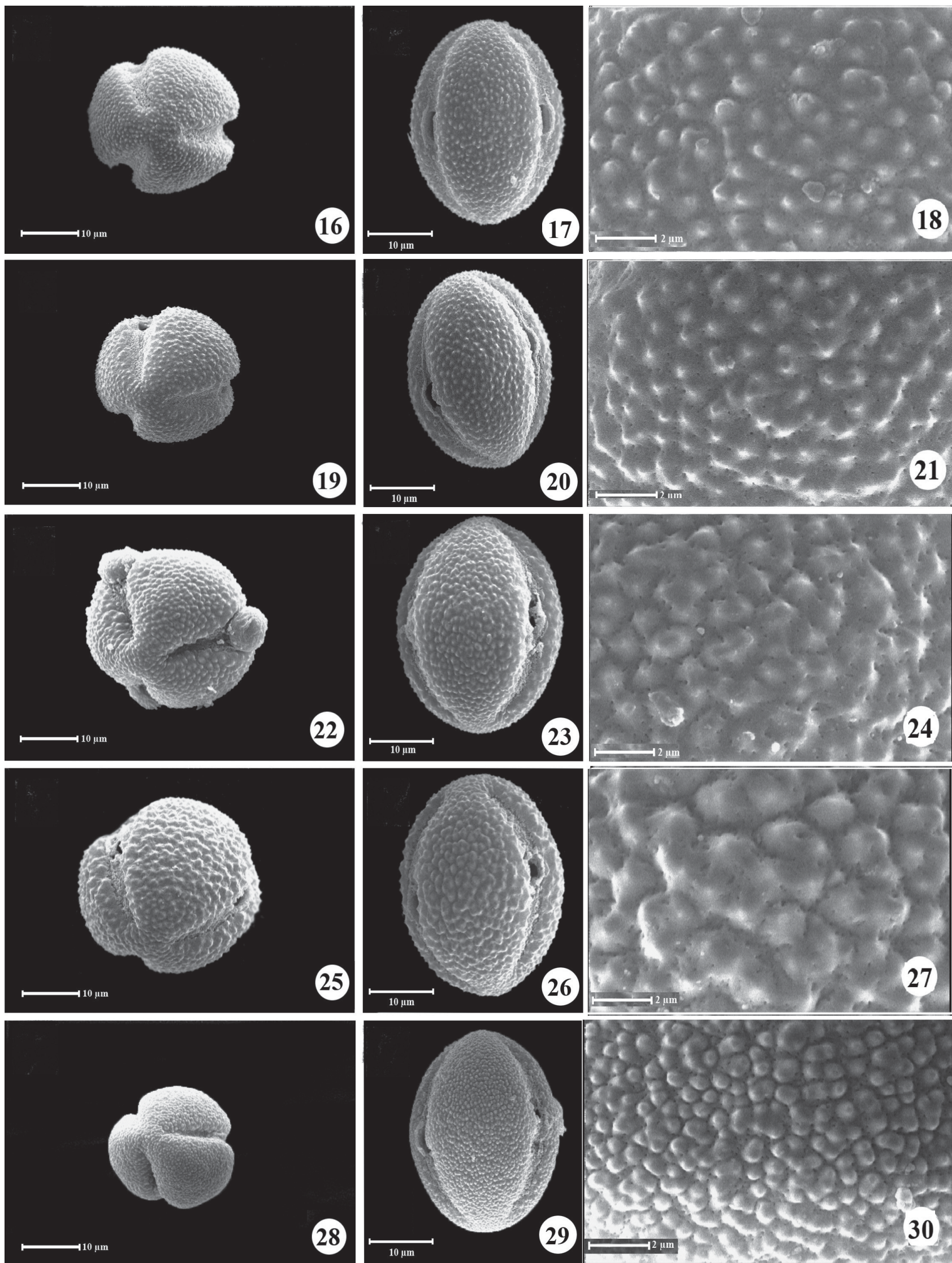

Figures 16-30. Pollen grains (SEM) of species of Centaurea L. (Asteraceae). (16-18) C. aucheri subsp. indistincta; (19-21) C. spectabilis; (22-24) C. amadanensis var. amadanensis; (25-27) C. amadanensis var. gymnoclada; (28-30) C. nemecii. 
Table 2. Size and shape of pollen grains of taxa of Centaurea L. (Asteraceae) examined. (PD - polar diameter; ED - equatorial diameter).

\begin{tabular}{|c|c|c|c|c|}
\hline Taxa & $\mathrm{PD} \mu \mathrm{m}$ & $\mathrm{ED} \mu \mathrm{m}$ & $\mathrm{PD} / \mathrm{ED}$ & Shape \\
\hline \multicolumn{5}{|l|}{ Sect. Cynaroides } \\
\hline C. gabrielae & $39.50(48.23 \pm 2.88) 53.02$ & $37.74(42.75 \pm 1.62) 45.12$ & 1.12 & prolate spheroidal \\
\hline C. geluensis & $39.34(45.21 \pm 3.96) 50.66$ & $37.40(42.48 \pm 3.01) 47.34$ & 1.06 & prolate spheroidal \\
\hline C. imperialis & $47.08(48.32 \pm 1.04) 49.78$ & $44.06(45.77 \pm 1.24) 47.20$ & 1.05 & prolate spheroidal \\
\hline C. phlomoides & $38.71(40.47 \pm 2.16) 43.36$ & $35.54(37.36 \pm 1.53) 39.00$ & 1.08 & prolate spheroidal \\
\hline C. regia subsp. regia & $42.09(50.36 \pm 4.61) 55.65$ & $38.12(48.35 \pm 4.60) 53.39$ & 1.04 & prolate spheroidal \\
\hline \multicolumn{5}{|l|}{ Sect. Phaeopappus } \\
\hline C. albonitens & $47.31(49.21 \pm 2.12) 52.76$ & $44.74(47.02 \pm 1.97) 48.49$ & 1.04 & prolate spheroidal \\
\hline C. aucheri subsp. elbursensis & $39.39(44.12 \pm 3.42) 47.81$ & $41.57(46.03 \pm 2.33) 50.25$ & 0.95 & oblate spheroidal \\
\hline C. aucheri subsp. farsistanica & $45.86(49.16 \pm 2.35) 52.53$ & $41.44(45.05 \pm 1.65) 47.65$ & 1.09 & prolate spheroidal \\
\hline C. aucheri subsp. indistincta & $39.19(44.05 \pm 4.06) 51.85$ & $37.17(39.31 \pm 1.51) 41.15$ & 1.12 & prolate spheroidal \\
\hline C. aucheri subsp. szowitsii & $37.29(41.31 \pm 1.26) 44.00$ & $36.19(38.28 \pm 1.41) 41.20$ & 1.07 & prolate spheroidal \\
\hline C. aucheri subsp. aucheri & $52.89(55.37 \pm 2.05) 58.98$ & $47.73(49.03 \pm 1.63) 52.68$ & 1.12 & prolate spheroidal \\
\hline C. spectabilis & $40.31(42.10 \pm 1.47) 45.15$ & $37.19(38.73 \pm 1.30) 42.15$ & 1.08 & prolate spheroidal \\
\hline \multicolumn{5}{|l|}{ Sect. Paraphysis } \\
\hline C. amadanensis var. amadanensis & $47.31(49.21 \pm 2.12) 52.76$ & $44.74(47.02 \pm 1.97) 48.49$ & 1.04 & prolate spheroidal \\
\hline C. amadanensis var. gymnoclada & $45.75(48.85 \pm 3.14) 56.61$ & $43.21(44.60 \pm 2.01) 49.28$ & 1.09 & prolate spheroidal \\
\hline C. nemecii & $43.44(47.50 \pm 2.61) 51.60$ & $39.86(41.45 \pm 1.31) 43.59$ & 1.14 & subprolate \\
\hline \multicolumn{5}{|l|}{ Sect. Microlophus } \\
\hline C. behen & $38.18(39.98 \pm 1.09) 41.09$ & $38.12(39.29 \pm 1.01) 40.17$ & 1.01 & prolate spheroidal \\
\hline C. koieana & $38.10(41.33 \pm 2.19) 44.52$ & $32.47(34.69 \pm 1.26) 37.26$ & 1.19 & prolate spheroidal \\
\hline C. pabotii & $37.55(41.11 \pm 2.14) 45.81$ & $32.38(36.25 \pm 1.70) 39.34$ & 1.13 & prolate spheroidal \\
\hline C. polypodiifolia var. polypodiifolia & $38.16(40.87 \pm 1.09) 43.05$ & $36.55(37.72 \pm 1.09) 39.23$ & 1.08 & prolate spheroidal \\
\hline
\end{tabular}

C. nemecii, to $0.5 / \mu \mathrm{m}^{2}$, in C. amadanensis var. gymnoclada. The distance between spines ranged from $0.27 \pm 0.1 \mu \mathrm{m}$, in $C$. aucheri subsp. farsistanica, to $1.31 \pm 0.58 \mu \mathrm{m}$, in C. gabrielae. Spine length was longest in $C$. albonitens $(0.85 \pm 0.22 \mu \mathrm{m})$ and shortest in C. aucheri subsp. aucheri $(0.23 \pm 0.08 \mu \mathrm{m})$.

On the basis of the exine ornamentation observed under SEM, we categorized the pollen grains into two types: type I, in which there are dense acute spinules on the exine surface, including C. albonitens, C. aucheri subsp. farsistanica, C. aucheri subsp. szowitsii, C. nemecii, C. phlomoides and C. regia subsp. regia (Fig. 3, 12, 15, 30, 54 and 57); and type II, in which such spinules are sparsely distributed on the exine surface, including C. gabrielae, C. koieana and C. polypodiifolia var. polypodiifolia (Fig. 36, 42 and 45). Subapical perforations in the spine base were observed in all of the taxa investigated (Fig. 3, 6, 9, 12, 15, 18, 21, 24, 27, 30, 33, $36,39,42,45,48,51,54$ and 57 ).

\section{Discussion}

In attempts to solve phylogenetic problems in Centaurea, the first detailed morphological analyses of pollen grains in the genus were made by Wagenitz (1955) and Avetisjan (1964). Wagenitz (1955) typified Centaurea pollen grains by morphological features, using the following features to group acetolyzed pollen: pollen shape, exine ornamentation, internal and external layers of columellae, length of colpus, pore shape and costae. Serrulata, Crupina and Centaurium pollen types were recognized as the most primitive; Dealbata, Cyanus, and Montana as intermediate; and Scabiosa and Jacea as the most advanced. Here we used some additional features such as the distance between colpi, the distance between a colpus and the pole, perforations $/ \mu \mathrm{m}^{2}$ and microspines $/ \mu \mathrm{m}^{2}$. The pollen grains of 19 of the taxa analyzed in the present study represent Wagenitz's Jacea 
Table 3. Pollen apertures data of taxa of Centaurea L. (Asteraceae) examined. (PW - pore width; CL - colpus length; CW - colpus width; CCD - colpus-colpus distance; CPD - colpus-pole distance).

\begin{tabular}{|c|c|c|c|c|c|c|}
\hline Taxa & $\mathrm{PW}(\mu \mathrm{m})$ & $\mathrm{CL}(\mu \mathrm{m})$ & $\mathrm{CW}(\mu \mathrm{m})$ & $\mathrm{CL} / \mathrm{CW}$ & $\operatorname{CCD}(\mu \mathrm{m})$ & $\mathrm{CPD}(\mu \mathrm{m})$ \\
\hline \multicolumn{7}{|l|}{ Sect. Phaeopappus } \\
\hline C. albonitens & $2.51(5.62 \pm 1.30) 7.57$ & $\begin{array}{c}49.97(52.49 \pm 1.66) \\
54.34\end{array}$ & $\begin{array}{c}8.95(11.08 \pm 1.78) \\
13.68\end{array}$ & 4.73 & $\begin{array}{c}31.37(33.63 \pm 1.64) \\
37.23\end{array}$ & $\begin{array}{c}18.20(19.46 \pm 1.25) \\
21.52\end{array}$ \\
\hline C. aucheri subsp. elbursensis & $2.37(4.16 \pm 1.77) 6.28$ & $\begin{array}{c}33.49(37.09 \pm 4.05) \\
43.37\end{array}$ & $\begin{array}{c}9.47(12.57 \pm 1.67) \\
14.52\end{array}$ & 2.95 & $\begin{array}{c}28.44(30.95 \pm 1.76) \\
33.68\end{array}$ & $\begin{array}{c}16.54(18.72 \pm 1.29) \\
21.14\end{array}$ \\
\hline C. aucheri subsp. farsistanica & $3.75(5.34 \pm 0.88) 5.79$ & $\begin{array}{c}45.99(48.58 \pm 2.12) \\
52.13\end{array}$ & $\begin{array}{c}6.68(10.26 \pm 2.86) \\
15.05\end{array}$ & 4.73 & $\begin{array}{c}28.95(31.88 \pm 1.97) \\
34.53\end{array}$ & $\begin{array}{c}15.44(18.49 \pm 1.59) \\
20.83\end{array}$ \\
\hline C. aucheri subsp. indistincta & $4.43(5.57 \pm 1.28) 7.64$ & $\begin{array}{c}33.51(37.67 \pm 2.76) \\
41.17\end{array}$ & $\begin{array}{c}6.56(10.70 \pm 4.28) \\
16.71\end{array}$ & 3.52 & $\begin{array}{c}24.25(26.78 \pm 1.52) \\
28.22\end{array}$ & $\begin{array}{c}13.60(16.38 \pm 2.16) \\
18.33\end{array}$ \\
\hline C. aucheri subsp. szowitsii & $1.92(3.14 \pm 0.74) 4.43$ & $\begin{array}{c}37.34(41.38 \pm 2.46) \\
43.37\end{array}$ & $5.30(7.53 \pm 1.27) 10.04$ & 5.49 & $\begin{array}{c}23.52(25.12 \pm 0.88) \\
26.52\end{array}$ & $\begin{array}{c}13.15(14.35 \pm 0.84) \\
15.89\end{array}$ \\
\hline C. aucheri subsp. aucheri & $4.61(6.93 \pm 1.47) 10.25$ & $\begin{array}{c}44.92(51.22 \pm 4.54) \\
56.12\end{array}$ & $\begin{array}{c}14.00(18.66 \pm 2.06) \\
21.90\end{array}$ & 2.74 & $\begin{array}{c}31.29(34.66 \pm 1.89) \\
36.23\end{array}$ & $\begin{array}{c}19.41(22.27 \pm 1.49) \\
25.90\end{array}$ \\
\hline C. spectabilis & $1.64(3.15 \pm 0.67) 4.33$ & $\begin{array}{c}36.56(38.79 \pm 2.06) \\
41.42\end{array}$ & $6.77(9.66 \pm 1.46) 11.34$ & 4.01 & $\begin{array}{c}24.09(26.66 \pm 1.54) \\
29.42\end{array}$ & $\begin{array}{c}13.39(16.04 \pm 1.20) \\
18.75\end{array}$ \\
\hline \multicolumn{7}{|l|}{ Sect. Cynaroides } \\
\hline C. gabrielae & $4.00(6.05 \pm 1.23) 8.42$ & $\begin{array}{c}47.12(52.07 \pm 3.86) \\
57.64\end{array}$ & $\begin{array}{c}7.87(10.81 \pm 1.60) \\
12.92\end{array}$ & 4.81 & $\begin{array}{c}26.25(30.97 \pm 3.19) \\
37.98\end{array}$ & $\begin{array}{c}15.21(18.55 \pm 2.32) \\
23.61\end{array}$ \\
\hline C. geluensis & $3.11(4.10 \pm 0.71) 5.41$ & $\begin{array}{c}38.02(45.35 \pm 4.50) \\
51.59\end{array}$ & $6.62(9.15 \pm 2.22) 13.66$ & 4.95 & $\begin{array}{c}27.31(30.95 \pm 2.62) \\
37.66\end{array}$ & $\begin{array}{c}15.86(18.54 \pm 1.73) \\
22.38\end{array}$ \\
\hline C. imperialis & $3.43(4.67 \pm 1.01) 5.92$ & $\begin{array}{c}40.66(44.11 \pm 3.42) \\
48.93\end{array}$ & $\begin{array}{c}8.80(10.63 \pm 1.59) \\
17.18\end{array}$ & 4.14 & $\begin{array}{c}28.82(30.58 \pm 1.22) \\
31.82\end{array}$ & $\begin{array}{c}16.44(18.02 \pm 1.17) \\
19.18\end{array}$ \\
\hline C. phlomoides & $1.56(2.93 \pm 1.08) 4.09$ & $\begin{array}{c}31.34(36.73 \pm 3.06) \\
38.94\end{array}$ & $6.39(7.17 \pm 0.71) 8.21$ & 5.12 & $\begin{array}{c}24.46(25.73 \pm 1.07) \\
27.17\end{array}$ & $\begin{array}{c}13.92(14.99 \pm 0.68) \\
15.53\end{array}$ \\
\hline C. regia subsp. regia & $3.75(6.25 \pm 2.09) 9.26$ & $\begin{array}{c}34.68(41.89 \pm 6.84) \\
50.61\end{array}$ & $6.50(9.77 \pm 1.62) 11.67$ & 4.28 & $\begin{array}{c}30.02(34.88 \pm 3.43) \\
40.90\end{array}$ & $\begin{array}{c}15.75(19.78 \pm 1.64) \\
22.85\end{array}$ \\
\hline \multicolumn{7}{|l|}{ Sect. Paraphysis } \\
\hline $\begin{array}{l}\text { C. amadanensis var. } \\
\text { amadanensis }\end{array}$ & $2.51(5.62 \pm 1.30) 7.57$ & $\begin{array}{c}49.97(52.49 \pm 1.66) \\
54.34\end{array}$ & $\begin{array}{c}8.95(11.08 \pm 1.78) \\
13.68\end{array}$ & 4.73 & $\begin{array}{c}33.19(37.11 \pm 2.09) \\
40.79\end{array}$ & $\begin{array}{c}18.39(22.09 \pm 1.62) \\
26.41\end{array}$ \\
\hline $\begin{array}{l}\text { C. amadanensis var. } \\
\text { gymnoclada }\end{array}$ & $3.70(5.57 \pm 1.17) 7.03$ & $\begin{array}{c}41.38(48.41 \pm 6.11) \\
56.55\end{array}$ & $5.01(6.96 \pm 1.31) 8.56$ & 6.95 & $\begin{array}{c}28.42(31.15 \pm 1.64) \\
33.51\end{array}$ & $\begin{array}{c}16.13(17.85 \pm 1.22) \\
21.59\end{array}$ \\
\hline C. nemecii & $5.90(7.73 \pm 1.45) 11.31$ & $\begin{array}{c}45.59(47.71 \pm 3.62) \\
55.52\end{array}$ & $\begin{array}{c}6.57(10.87 \pm 3.72) \\
15.44\end{array}$ & 4.38 & $\begin{array}{c}25.43(29.06 \pm 1.92) \\
32.12\end{array}$ & $\begin{array}{c}17.56(19.42 \pm 1.25) \\
21.09\end{array}$ \\
\hline \multicolumn{7}{|l|}{ Sect. Microlophus } \\
\hline C. behen & $2.51(4.49 \pm 0.70) 5.66$ & $\begin{array}{c}32.35(36.18 \pm 3.53) \\
39.97\end{array}$ & $6.58(8.78 \pm 1.35) 11.64$ & 4.07 & $\begin{array}{c}25.30(26.92 \pm 1.05) \\
28.83\end{array}$ & $\begin{array}{c}14.15(16.16 \pm 1.10) \\
18.33\end{array}$ \\
\hline C. koieana & $1.64(4.14 \pm 1.25) 6.49$ & $\begin{array}{c}38.24(39.85 \pm 1.28) \\
41.60\end{array}$ & $6.91(9.97 \pm 1.68) 11.49$ & 3.99 & $\begin{array}{c}20.00(22.35 \pm 1.31) \\
23.59\end{array}$ & $\begin{array}{c}10.76(13.56 \pm 1.49) \\
15.62\end{array}$ \\
\hline C. pabotii & $2.63(4.16 \pm 1.03) 6.25$ & $\begin{array}{c}31.30(38.50 \pm 5.76) \\
45.82\end{array}$ & $\begin{array}{c}7.89(10.68 \pm 1.41) \\
12.57\end{array}$ & 3.60 & $\begin{array}{c}21.19(22.39 \pm 1.10) \\
25.79\end{array}$ & $\begin{array}{c}11.40(13.37 \pm 1.02) \\
15.40\end{array}$ \\
\hline $\begin{array}{l}\text { C. polypodiifolia var. } \\
\text { polypodiifolia }\end{array}$ & $2.08(3.66 \pm 0.73) 5.03$ & $\begin{array}{c}34.94(37.09 \pm 2.58) \\
41.53\end{array}$ & $5.92(9.31 \pm 1.50) 12.61$ & 3.98 & $\begin{array}{c}23.88(26.14 \pm 1.67) \\
31.61\end{array}$ & $\begin{array}{c}13.42(15.35 \pm 0.90) \\
17.17\end{array}$ \\
\hline
\end{tabular}


Table 4. Exine data pollen grains of taxa of Centaurea L. (Asteraceae) examined. (Ex - exine thickness; Per.n -perforations/ $\mu \mathrm{m}^{2}$; SL - spine length; SD - spine-spine distance); Ms.n - microspines $/ \mu^{2}{ }^{2}$; S - shape of pollen; Orn. - ornamentation (exine sculpture); $\mathrm{S}$ - scabrate).

\begin{tabular}{|c|c|c|c|c|c|c|}
\hline Taxa & $\operatorname{Ex}(\mu \mathrm{m})$ & Per.n & $\mathrm{SL}(\mu \mathrm{m})$ & $\mathrm{SD}(\mu \mathrm{m})$ & Ms.n & Orn. \\
\hline \multicolumn{7}{|l|}{ Sect. Phaeopappus } \\
\hline C. albonitens & $5.02(6.97 \pm 1.00) 7.89$ & 6.69 & $0.63(0.85 \pm 0.22) 0.95$ & $0.53(0.69 \pm 0.17) 0.75$ & 1.00 & $S$ \\
\hline C. aucheri subsp. elbursensis & $3.72(5.24 \pm 0.82) 7.24$ & 10.82 & $0.35(0.53 \pm 0.09) 0.59$ & $0.63(0.72 \pm 0.23) 0.81$ & 2.50 & $S$ \\
\hline C. aucheri subsp. farsistanica & $5.02(7.02 \pm 1.04) 8.58$ & 16.39 & $0.28(0.34 \pm 0.04) 0.39$ & $0.22(0.27 \pm 0.70) 0.31$ & 4.00 & S \\
\hline C. aucheri subsp. indistincta & $4.05(5.59 \pm 0.96) 6.53$ & 19.71 & $0.38(0.49 \pm 0.11) 0.57$ & $0.73(0.82 \pm 0.24) 0.89$ & 2.00 & $S$ \\
\hline C. aucheri subsp. szowitsii & $4.61(5.46 \pm 0.61) 6.58$ & 13.27 & $0.32(0.54 \pm 0.04) 0.58$ & $0.30(0.49 \pm 0.10) 0.55$ & 2.50 & S \\
\hline C.aucheri subsp. aucheri & $3.62(5.02 \pm 1.33) 5.35$ & 8.12 & $0.19(0.23 \pm 0.08) 0.29$ & $0.52(0.61 \pm 0.81) 0.69$ & 1.00 & S \\
\hline C. spectabilis & $5.35(6.07 \pm 0.63) 7.12$ & 10.82 & $0.44(0.51 \pm 0.06) 0.58$ & $0.59(0.63 \pm 0.36) 0.69$ & 2.50 & $S$ \\
\hline \multicolumn{7}{|l|}{ Sect. Cynaroides } \\
\hline C. gabrielae & $5.42(7.81 \pm 1.48) 10.37$ & 16.99 & $0.50(0.57 \pm 0.09) 0.68$ & $1.25(1.31 \pm 0.58) 1.43$ & 1.00 & S \\
\hline C. geluensis & $3.58(6.70 \pm 1.26) 8.12$ & 13.17 & $0.50(0.58 \pm 0.19) 0.67$ & $0.71(0.78 \pm 0.19) 0.84$ & 1.50 & S \\
\hline C. imperialis & $5.85(7.00 \pm 0.83) 8.11$ & 13.23 & $0.30(0.37 \pm 0.05) 0.42$ & $0.46(0.53 \pm 0.17) 0.58$ & 3.00 & S \\
\hline C. phlomoides & $4.72(5.44 \pm 0.45) 5.90$ & 10.93 & $0.42(0.51 \pm 0.04) 0.58$ & $0.60(0.68 \pm 0.30) 0.75$ & 2.00 & S \\
\hline C. regia subsp. regia & $4.60(7.03 \pm 1.35) 9.23$ & 10.44 & $0.48(0.59 \pm 0.08) 0.62$ & $0.45(0.52 \pm 0.23) 0.58$ & 3.00 & $S$ \\
\hline \multicolumn{7}{|l|}{ Sect. Paraphysis } \\
\hline C. amadanensis var. amadanensis & $4.55(6.38 \pm 1.20) 8.09$ & 12.26 & $0.42(0.49 \pm 0.15) 0.55$ & $0.38(0.46 \pm 0.15) 0.53$ & 1.44 & $S$ \\
\hline C. amadanensis var. gymnoclada & $5.14(6.12 \pm 0.60) 7.64$ & 9.86 & $0.81(0.83 \pm 0.12) 0.87$ & $0.51(0.57 \pm 0.17) 0.62$ & 0.50 & S \\
\hline C. nemecii & $5.90(7.44 \pm 1.09) 8.91$ & 17.43 & $0.21(0.29 \pm 0.04) 0.35$ & $0.27(0.32 \pm 0.10) 0.39$ & 5.50 & $S$ \\
\hline \multicolumn{7}{|l|}{ Sect. Microlophus } \\
\hline C. behen & $3.97(4.58 \pm 0.43) 5.12$ & 16.54 & $0.32(0.36 \pm 0.020) 0.43$ & $0.70(0.73 \pm 0.13) 0.80$ & 2.00 & $S$ \\
\hline C. koieana & $3.24(4.52 \pm 0.63) 5.35$ & 9.00 & $0.29(0.39 \pm 0.070) 0.42$ & $0.90(0.99 \pm 0.36) 1.08$ & 1.00 & S \\
\hline C. pabotii & $3.96(5.98 \pm 1.01) 7.42$ & 14.97 & $0.45(0.51 \pm 0.080) 0.56$ & $0.61(0.65 \pm 0.18) 0.72$ & 2.00 & S \\
\hline C. polypodiifolia var. polypodiifolia & $4.52(5.75 \pm 0.90) 6.75$ & 5.04 & $0.30(0.36 \pm 0.03) 0.45$ & $1.20(1.24 \pm 0.19) 1.32$ & 2.00 & $S$ \\
\hline
\end{tabular}

pollen type, based on the following characters: spheroidal subprolate, scabrate ornamentation and long colpus. Wagenitz \& Hellwig (2000) identified that same pollen type in several Centaurea sections occurring in eastern Turkey, the Caucasus and northwest Iran.

The Cyanus and Montana pollen types are limited to Centaurea Sect. Cyanus, and the Scabiosa type is characteristic of section Acrocentron in its broadest sense (Garcia-Jacas et al. 2000). Avetisjan (1964) divided the Jacea type into six subgroups based on morphological variations in this pollen type. The author also combined the Dealbata, Montana and Cyanus types into one large group, Psephellus. In the Jacea pollen type, the spines are reduced are subprolate spheroidal shape. Wagenitz $(1955,1976)$ put forward the idea that reduction of the spines and inner columellae, lengthening of the colpus and thickening of the costae are progressive characteristics in the Asteraceae family. In all taxa studied here, the spines are shorter than $1 \mu \mathrm{m}$ and the colpus are quite long. An operculum found near the aperture is accepted as a progressive evolutionary characteristic (Pehlivan 1994). Skvarla et al. (1977) and Stix (1960) claimed that subapical perforations in the spine base in Asteraceae are a good taxonomic character for discriminating taxa. Subapical perforations in the spine base were observed in all of the taxa investigated here.

In the present study, pollen characters (pollen shape, exine structure and sculpturing) proved to be useful characters for classification. Further morphological, karyological, palynological and molecular studies will help resolve problems related to the taxonomy of Centaurea. 

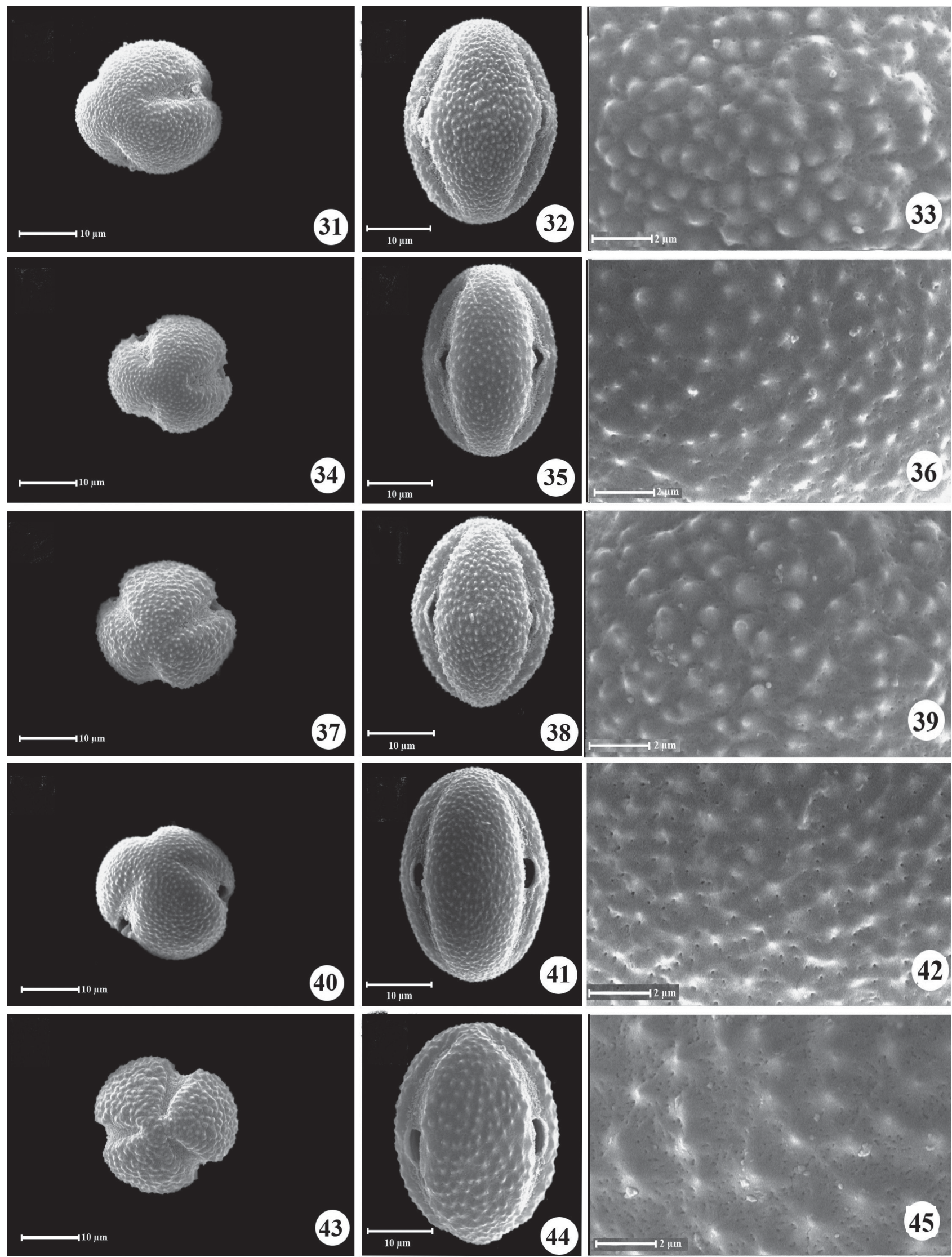

Figures 31-45. Pollen grains (SEM) of species of Centaurea L. (Asteraceae). (31-33) C. behen; (34-36) C. koieana; (37-39) C. pabotii; (40-42) C. polypodiifolia var. polypodiifolia; (43-45) C. gabrielae. 

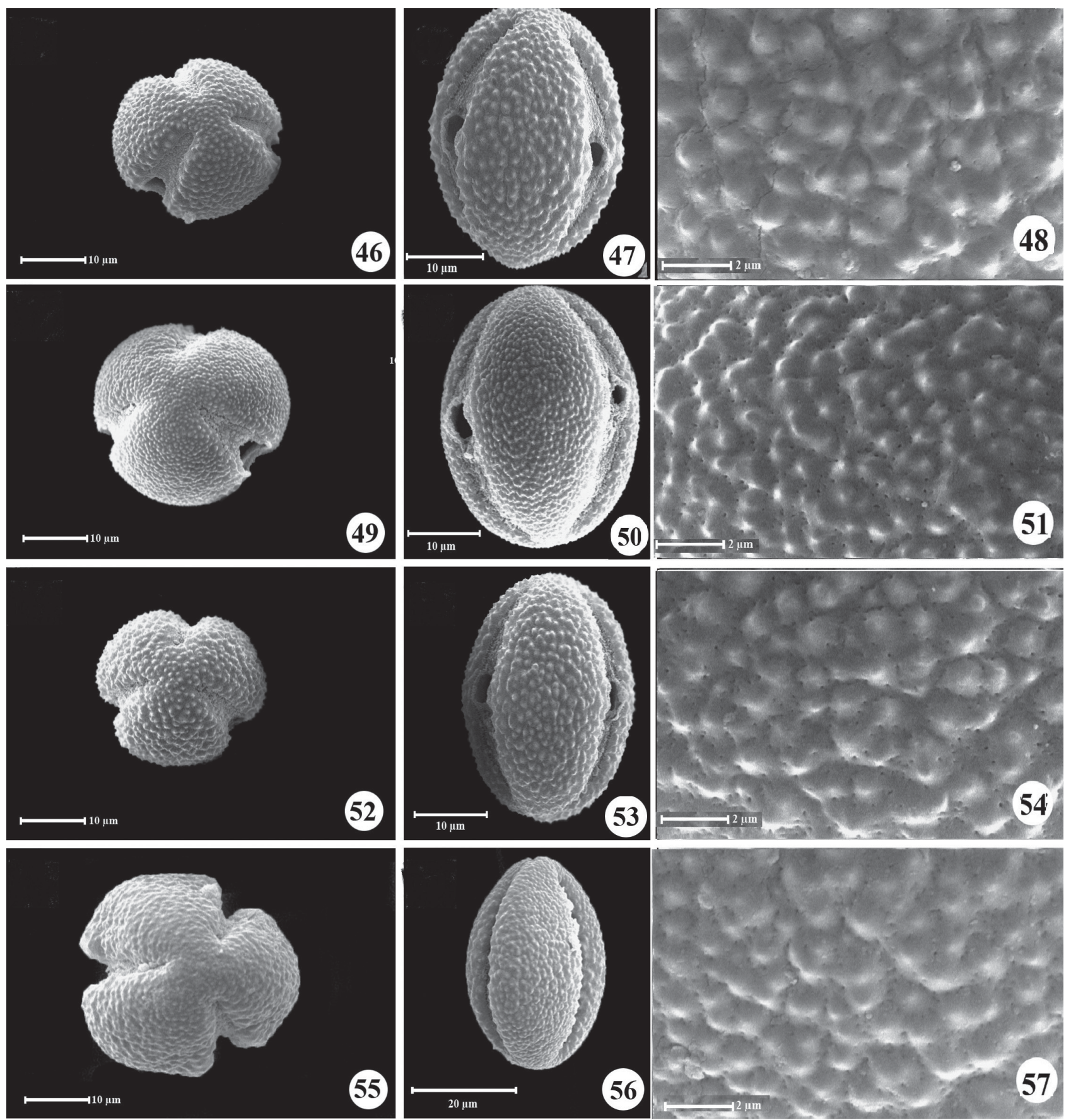

Figures 46-57. Pollen grains (SEM) of species of Centaurea L. (Asteraceae). (46-48) C. geluensis; (49-51) C. imperialis; (52-54) C. phlomoides; (55-57) C. regia subsp. regia.

\section{Acknowledgments}

We thank Dr. Farideh Attar and Dr. Mohammad Amini Rad, curators of the Central Herbarium of the University of Tehran and the Herbarium of the Iranian Research Institute of Plant Protection, respectively. We are also grateful to Rezaei (University of TarbiatModares) for his kind assistance in taking the SEM photomicrographs.

\section{References}

Avetisjan, E.M. 1964. Palynolosystematique de la tribu des Centaureinae des Asteraceae. Trudy Botanicheskogo Institute Akademii Nauk Armajansk 14: 31-47.

Celik, S.; Uysal, I.; Menemen, Y. \& Karabacak, E. 2005. Morphology, Anatomy, Ecology, Pollen and Achene structure of Centaurea consanguinea DC. (Sect. Acrolophus). International Journal of Botany 1(1): 85-89.

Celik, S.; Uysal, I. \& Menemen, Y. 2008. Morphology, Anatomy, Ecology and Palynology of two Centaurea species from Turkey. Bangladesh Journal of Botany 37(1): 67-74. 

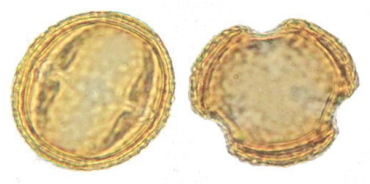

58

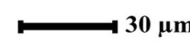

61
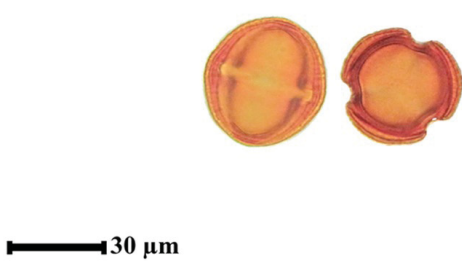

59
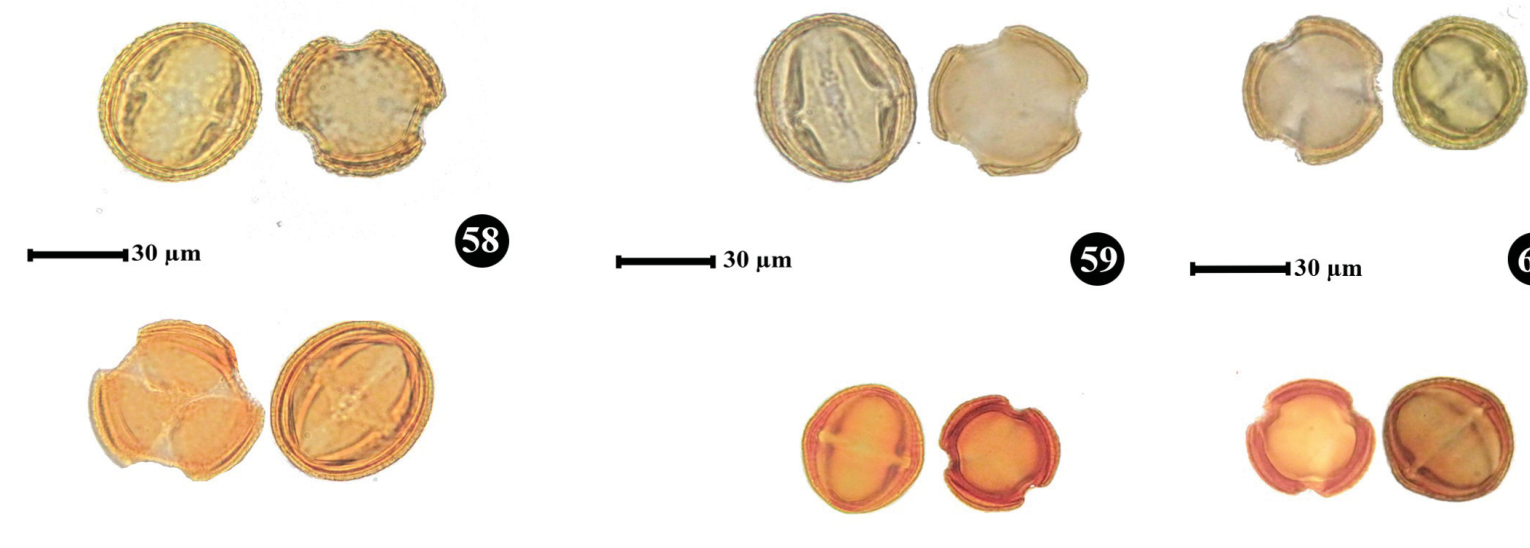

$30 \mu \mathrm{m}$
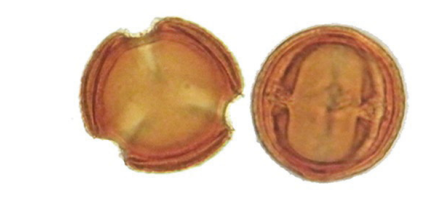

$30 \mu \mathrm{m}$
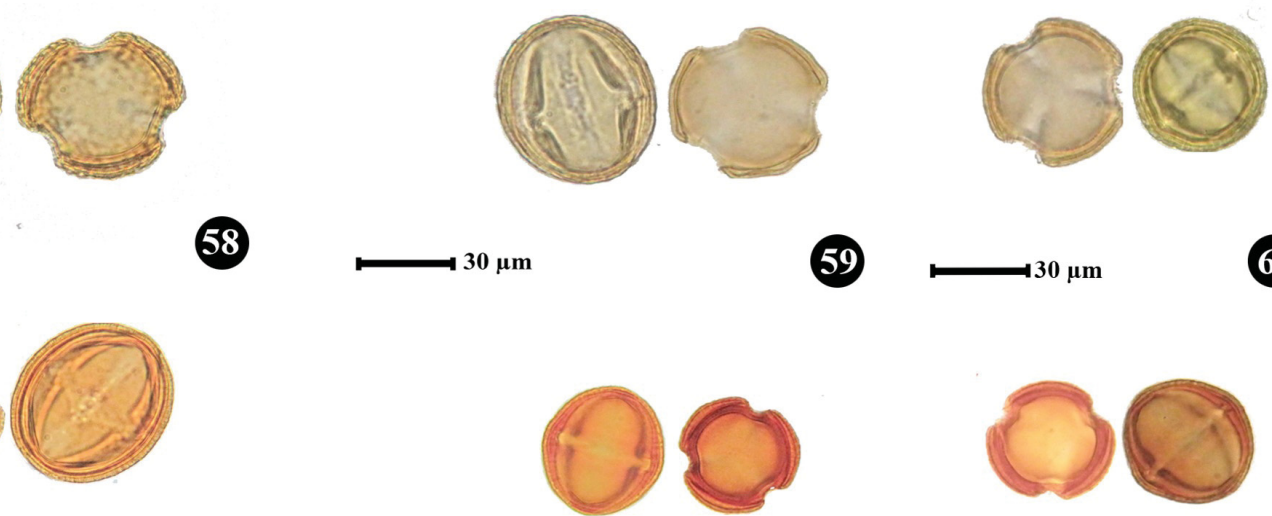

62
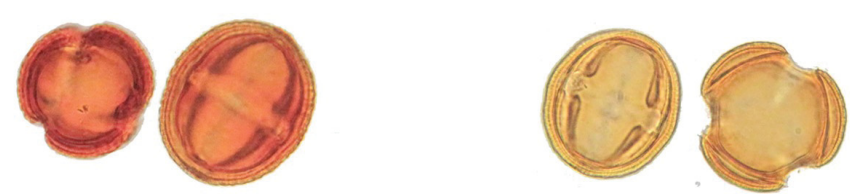

64

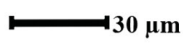

65

60

63

66

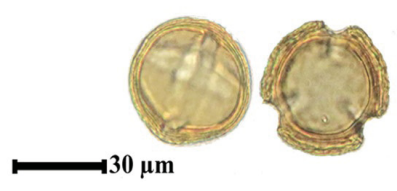

67

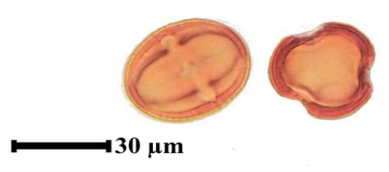

68
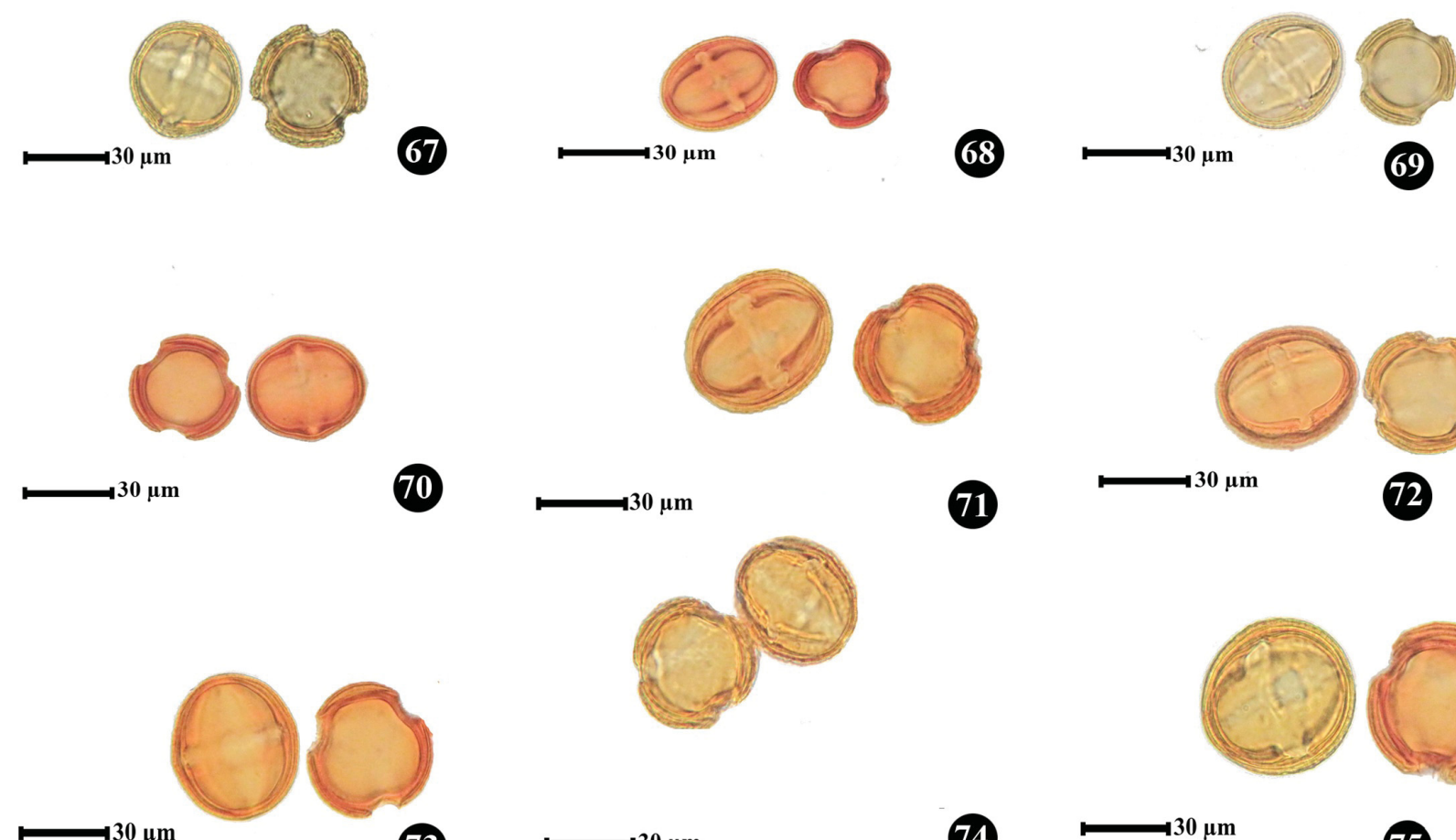

70

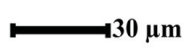

71
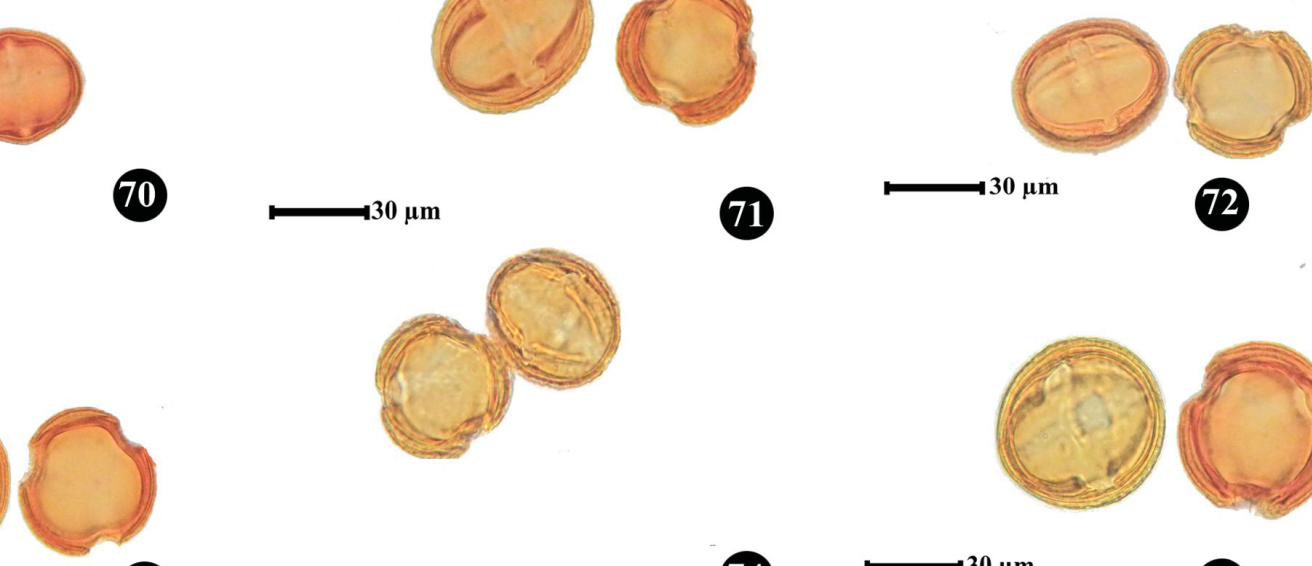

73

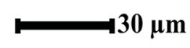

74
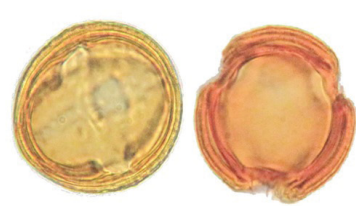

75

Figures 58-75. Pollen grains (LM) of species of Centaurea L. (Asteraceae). (58) C. albonitens; (59) C. aucheri subsp. aucheri; (60) C. aucheri subsp. elbursensis; (61) C. aucheri subsp. farsistanica; (62) C. aucheri subsp. szowitsii; (63)C. spectabilis; (64) C. amadanensis var. amadanensis; (65) C. amadanensis var. gymnoclada; (66) C. nemecii; (67) C. behen; (68) C. koieana (69) C. pabotii; (70) C. polypodiifolia var. polypodiifolia; (71) C. gabrielae; (72) C. geluensis; (73) C. imperialis; (74) C. phlomoides; (75) C. regia subsp. regia. 
Davis, P.H.; Mill, R. \& Tan K. (Eds). 1988. Flora of Turkey and the East Aegean Islands, (Supplement) 10: 489-501. Edinburgh, Edinburgh University Press.

Erdtman, G. 1952. Pollen Morphology and Plant Taxonomy. Stockholm, Almqvist \& Wiksell.

Erdtman, G. 1960. Acetolysis method, a revised description. Svensk Botanisk Tidskrift 54: 561-564.

Garcia-Jacas N.; Susanna, A.; Mozaffarian, V. and Ilarslan, R. 2000. The natural delimitation of Centaurea (Asteraceae, Cardueae): ITS sequence Analysis of the Centaurea jacea group. Plant Systematic and Evolution 223: 185-199.

Ghahreman, A. \& Attar, F. 1999. Biodiversity of plant species in Iran. Tehran, Tehran University Publications.

Halbritter, H.; Werber, M.; Zetter, R. \& Frosch-Radivo, A.; Buchner, R. \& Hesse, M. 2007. Illustrated Handbook on Pollen Terminology. http://www.paldat.org/paldat-terminolgy-large.pdf.

Heywood, V.H. 1979. Flowering plants of the world. Oxford, Oxford University Press.

Hickey, M. \& King, C. J. 1981. 100 families of flowering plants. Cambridge, Cambridge University Press.

Jaffari, E. \& Ghanbarian, G. 2007. Pollen morphological studies on selected taxa of Asteraceae. Journal of Plant Science 2(2): 195-201.

Moore, P.D.; Webb, J.A. \& Collinson, M.E. 1991. Pollen analysis. 2nd. ed. City, Black-Well Scientific publications.

Özler, H.; Kaya, Z. \& Pehiivan, S. 2009. Pollen morphology of some Centaurea L., Psephellus Cass. and Cyanus Miller taxa. Acta Biologica Cracoviensia, Series Botanica 51(2): 53-66.

Pehlivan, S. 1994. Scanning Electron Microscope studies of the pollen grains if some Turkish endemic Centaurea. Journal of Faculty of Pharmacy of Gazi University 11 (2): 205-211.
Rendle, A.B. 1976. The classification of flowering plants. 2v. Cambridge, Cambridge University Press.

Skvarla, J...; Turner, B.L.; Paltel, V.C. \& Tobb, A.S. 1977. Pollen Morphology in the Compositae and in morphologically related families. Pp. 141248. In: Heywood, V.H.; Harborne, J.B. and Turner, B.L. (Eds.). The Biology and Chemistry of the Compositae. London, Academic Press.

Stix E. 1960. Pollen Morphologische untersuchungen an Compisiten. Grana Palynologica 2: 41-104.

Uysal, I.; Celik, S. \& Menemen, Y. 2005. Morphology, Anatomy, Ecology, Pollen and Achene Features of Centaurea polyclada DC. (Sect. Acrolophus) in Turkey. Journal of Biological Sciences 5(2): 176-180.

Villodre, J.M. \& Gracia-Jacas N. 2000. Pollen Studies in Subtribe Centaureinae (Asteraceae): the Jacea group analyzed with Electron Microscopy. Botanical Journal of Linnean Society 133(4): 473-484.

Wagenitz, G. 1955. Pollen Morphologie und Systematik in der Gattung Cenntaurea weldeniana (Asteraceae). s.l. Flora 142: 213-279.

Wagenitz, G. 1976. Systematics and Phylogeny of the Compositae (Asteraceae). Plant Systematic and Evolution 125: 29-46.

Wagenitz, G. 1980. Centaurea. In: Rechinger, K.H. (Ed.). Flora Iranica, Lieferung 139b: 313-420.

Wagenitz, G. 1986. Centaurea in south-west Asia: Patterns of distribution and diversity. Proceeding Royal Society Edinburgh 89: 11-21.

Wagenitz, G. \& Hellwig, F. 2000. The genus Psephellus Cass. (Compositae, Cardueae) revisited with a broadened concept. Willdenowia 30: 29-44.

Wodhouse, R.P. 1935. Pollen grains. New York, McGraw Hill Press.

Zamani, A.; Attar, F. \& Maroofi, H. 2010. Pollen morphology of the genus Pyrus L. (Rosaceae) in Iran. Acta Biologica Szegediensis 54(1): 1-6. 